\title{
Tuning the Friction of Graphene on Mica by Alcohol Intercalation
}

\author{
Edwin Dollekamp, ${ }^{* \dagger}$ † Pantelis Bampoulis, ${ }^{\ddagger}$ Martin H. Siekman, E. Stefan Kooij, \\ and Harold J. W. Zandvliet* \\ Physics of Interfaces and Nanomaterials, MESA+ Institute for Nanotechnology, University of Twente, P.O. Box 217, 7500 AE \\ Enschede, The Netherlands
}

\section{Supporting Information}

ABSTRACT: The friction of graphene on mica was studied using lateral force microscopy. We observed that intercalation of alcohol molecules significantly increases the friction of graphene, as compared to water. An increase of 1.8, 2.4, and 5.9 times in friction between the atomic force microscopy tip and single-layer graphene was observed for methanol, ethanol, and 2-propanol, respectively. Moreover, the friction of graphene is found to be higher for single-layer graphene than for multilayer graphene. We attribute the increase in friction to the additional vibrational modes of alcohol molecules. The significant variation of the frictional character-

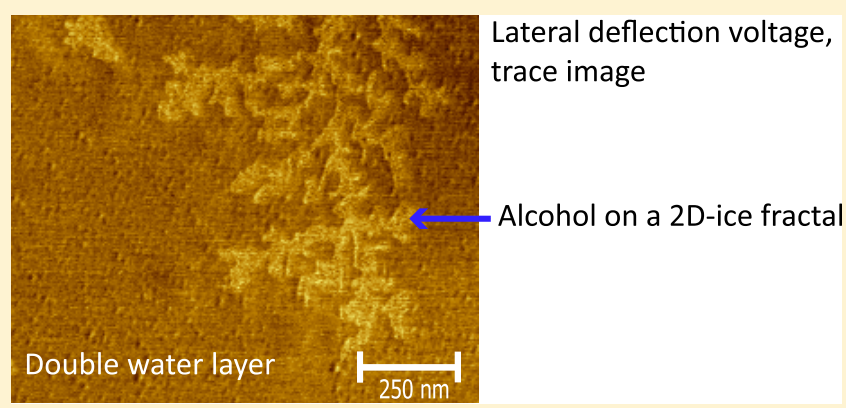
istics of graphene at the nanoscale by altering the intercalant could open up applications for the next-generation nanolubricants and nanodevices.

\section{INTRODUCTION}

Friction is the process when translational kinetic energy is converted to the surface atom vibrations. ${ }^{1}$ For example, in atomic force microscopy (AFM), friction results in the transfer of momentum from the tip to the surface atoms. The control over friction at the nanoscale is of great importance for nanomechanical applications in tribology. ${ }^{2-5}$ In most cases, a lower friction is favorable due to lower energy losses, e.g., in lubricant systems. On the other hand, an increase in friction is sometimes desirable, e.g., in braking systems. Having the possibility to tune the friction between a high and low state could result in novel nanodevices.

An interesting system to investigate the friction of supported two-dimensional (2D) materials consists of graphene on mica, between which molecules can intercalate from the vapor phase. For graphene on mica, the excitation and energy transfer process can be studied separately. When an AFM tip slides over a graphene surface, it excites out-of-plane lattice vibrations of graphene, the so-called flexural phonons. ${ }^{6}$ This lattice vibrational energy is transferred to the out-of-plane phonon modes of the intercalated molecules and the mica. Lee et al. ${ }^{7}$ showed that increasing the number of intercalated water layers between graphene and mica resulted in a higher friction on the graphene. They attributed the higher friction to the increase of the out-of-plane phonon density of states (DOS) for the graphene and the water, and the larger overlap of the phonon DOS between graphene, water, and mica. They also found that when replacing $\mathrm{H}_{2} \mathrm{O}$ molecules with $\mathrm{D}_{2} \mathrm{O}$, the friction was reduced due to the larger mass of the $\mathrm{D}_{2} \mathrm{O}$ molecules, which resulted in a lower phonon density of states frequency and thus a lower energy dissipation. ${ }^{8}$
In this work, we study whether the friction of graphene can be tuned by changing the size and therewith the vibrational modes of the intercalated molecules. For this, we replaced water by larger alcohol molecules. The alcohol molecules have different vibrational modes, e.g., the stretching of the $\mathrm{C}-\mathrm{C}$ bond and the $\mathrm{C}-\mathrm{O}$ bonds. We also investigated whether the friction of graphene can be tuned by varying the size of the intercalated alcohol molecules. For this purpose, we used methanol, ethanol, and 2-propanol. Moreover, in this study, we also addressed the effect of graphene layer thickness on the friction.

\section{EXPERIMENTAL SECTION}

The mechanical exfoliation method was used to deposit graphene on mica. $^{9-11}$ We have used muscovite mica (SPI, V1) as a substrate. Mica is a mineral that can be cleaved to atomically flat layers. ${ }^{12}$ Graphene is a single-atom-thin 2D material that is flexible and impermeable to water and gases. ${ }^{13,14}$ Water can easily intercalate between the two materials, since mica is hydrophilic. ${ }^{15}$ During the mechanical exfoliation method, a piece of highly oriented pyrolytic graphite (HOPG, grade ZYB, MikroMasch) was cleaved with Scotch tape. After cleavage, loose vertical standing flakes were transferred with a tweezer from the HOPG substrate to the mica sample. By not using Scotch tape for the HOPG transfer, contamination due to tape was prevented. ${ }^{16}$ The HOPG flake was then gently pressed onto the mica and subsequently removed. In some cases, small HOPG flakes remained attached to the mica surface. The small graphene flakes on mica were located by optical reflection microscopy (Leica DM2500 $\mathrm{MH}) \cdot{ }^{17,18}$ In this setup, the light source and microscope objective were both positioned above the sample. ${ }^{19}$ The thickness of the

Received: February 17, 2019

Published: March 18, 2019 

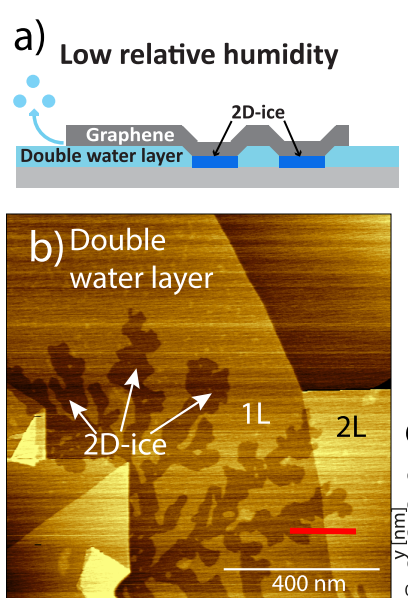

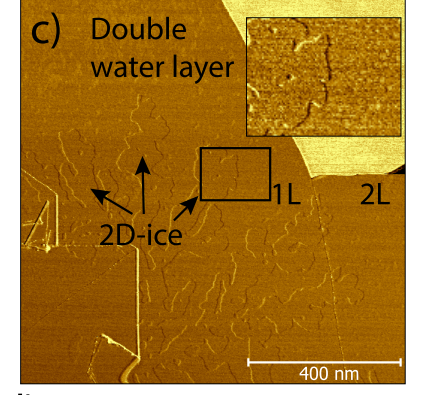

d)

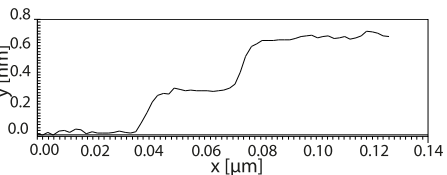

Figure 1. (a) Schematic illustration of the sample under low-humidity conditions. (b) An AFM topography image under low relative humidity conditions, relative humidity $(\mathrm{RH}) \sim 0.1 \%$. (c) A simultaneously obtained AFM friction image (lateral deflection voltage, trace image). (d) Height profile of the red line in (b).

graphene flakes was further characterized by AFM (Agilent 5100), which was equipped with an environmental chamber that allows exposure of the sample to different gases. To obtain lateral force microscopy (LFM) images, the AFM was operated in contact mode.

The AFM environmental chamber was initially purged with nitrogen to remove water vapor. Subsequently, we started purging with alcohol vapor. The alcohol was placed in a gas washing bottle, which was already in place during the purging with nitrogen to further reduce the water concentration of the system. We quickly opened the gas washing bottle and filled it approximately halfway with alcohol. The nitrogen flow rate was adjusted to $\sim 0.5 \mathrm{~L} / \mathrm{min}$, such that there was only calm bubbling of alcohol in the gas washing bottle. In our experiments, we used three different alcohols: methanol (SigmaAldrich, anhydrous 99.8\%), ethanol (EMSURE, analytical quality), and 2-propanol (Sigma-Aldrich, anhydrous, 99.5\%). The alcohols must have a water concentration as low as possible. Having water contamination in the alcohols results in the filling of the fractals with water instead of alcohol. ${ }^{20}$ We established a low water concentration by using anhydrous alcohols. Furthermore, we used a new bottle of alcohol for every measurement, since after opening, water from the ambiance enters the bottle that contaminates alcohol. The measurements are repeated several times for every alcohol to confirm the reproducibility of the friction observations.

In the measurement of Figures 1, 2, 3, and 5, we used a AD-E-0.5SS tip (diamond tip, Adama Innovations) with a nominal spring constant of $0.5 \mathrm{~N} / \mathrm{m}$ and a nominal resonance frequency of $30 \mathrm{kHz}$. This AFM tip is very sharp, which allows to visualize individual potassium ions on mica. ${ }^{21}$ In the measurement of Figure 4, we used a PPP-CONTSCR AFM tip (NANOSENSORS), with a nominal spring constant of $0.2 \mathrm{~N} / \mathrm{m}$ and a nominal resonance frequency of $13 \mathrm{kHz}$. This AFM tip is well-suited for lateral force microscopy, since it has a flexible cantilever, resulting in a high lateral sensitivity.

To convert the lateral deflection voltage to a lateral force, the improved wedge calibration method of Varenberg et al. ${ }^{22}$ was used. This method is based on the wedge calibration method of Ogletree et al. $^{23}$ In the improved wedge calibration method, a TGF11 silicon calibration grating (MikroMasch), with trapezoidal steps under an angle of $54.74^{\circ}$, was scanned. In the Supporting Information, details of this calibration method are provided.

\section{RESULTS AND DISCUSSION}

The graphene-mica sample was first scanned under ambient conditions and at room temperature. Under ambient conditions, water is always present between the two materials. $^{15,24-30}$ There is still a discussion in literature about how many layers are intercalated between graphene and mica
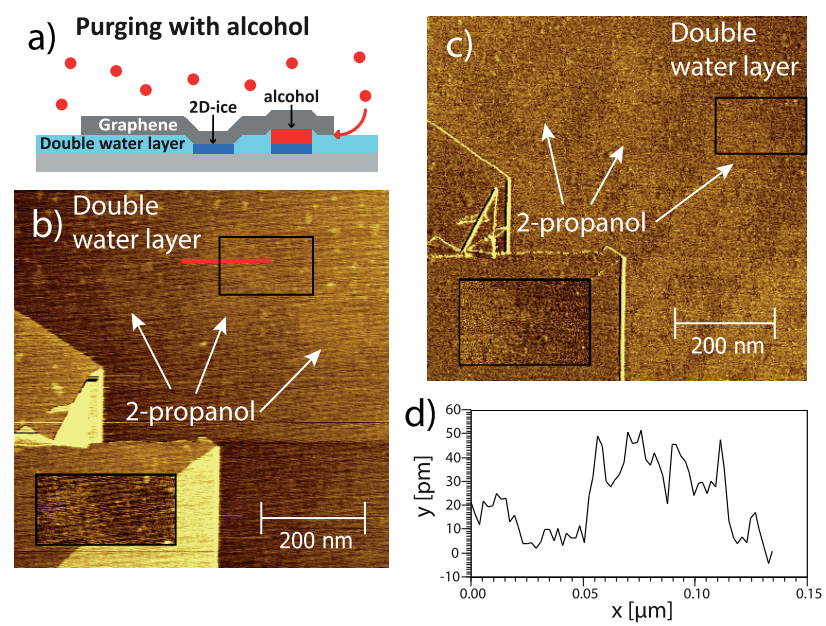

Figure 2. (a) Schematic illustration of the sample when purged with alcohol. (b) An AFM topography image. The sample was exposed to a 2-propanol environment. The fractal filled with 2-propanol. (c) Simultaneously obtained AFM friction image (lateral deflection voltage, trace image). An AD-E-0.5-SS AFM tip was used. (d) Height profile of the red line in (b).
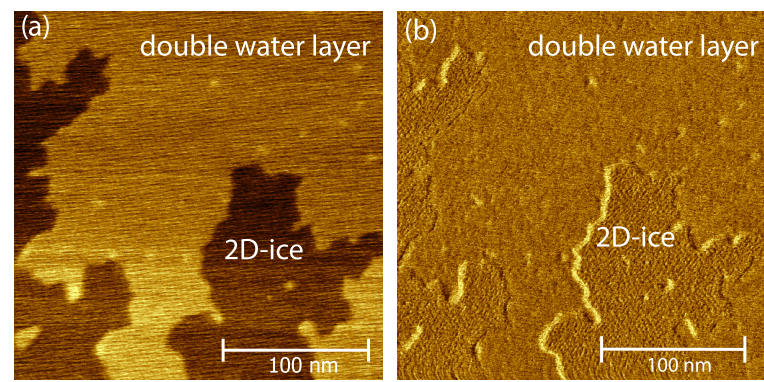

Figure 3. (a) AFM topography image under low RH conditions. (b) The simultaneously obtained AFM friction image (lateral deflection voltage, trace image). The potassium ions can be observed at the location of the 2D-ice. An AD-E-0.5-SS AFM tip was used.

under ambient conditions. In the structural model developed by Bampoulis et al., which we use in this paper, two water layers are located between graphene and mica under ambient 


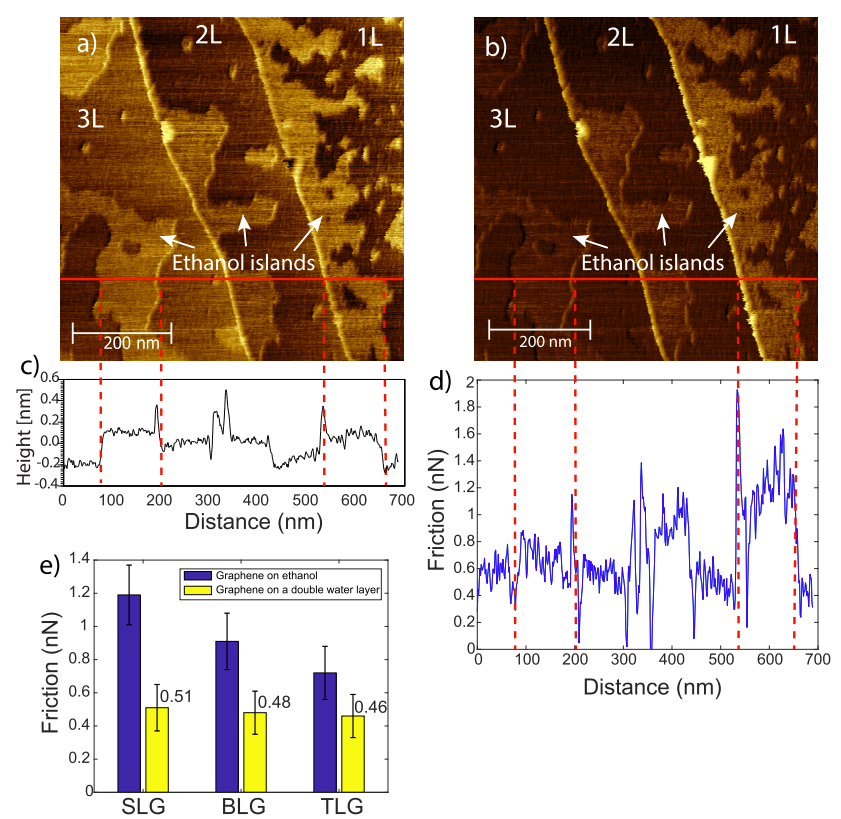

Figure 4. (a) AFM topography image. The brighter regions represent intercalated ethanol islands. A PPP-CONTSCR AFM tip was used. (b) The simultaneously obtained AFM friction image (lateral deflection voltage, trace image). The normal force was $66.4 \mathrm{nN}$. (c) Line profile of the height, corresponding to the red line in (a). (d) Line profile of the friction, corresponding to the red line in (b). (e) Bar plot of the friction of graphene on ethanol and the double water layer for single-layer graphene (SLG), bilayer graphene (BLG), and trilayer graphene (TLG).

conditions. ${ }^{21,31-33}$ When lowering the humidity, the top water layer evaporates out of the slit pore and fractal-like structures appear. The fractals have a clear crystalline shape. When pressure is applied with an AFM tip, a contrast appears inside the fractals. ${ }^{34}$ This contrast is visible in the topography and friction images. The authors explain this contrast by melting of the crystalline water to a quasi-liquid phase. Therefore, a water layer needs to be present in the fractal. Since the fractal shows a higher conductivity with C-AFM, the water is a monolayer and has a crystalline orientation which p-type dopes the graphene. This p-type doping has also been observed by scanning tunneling microscopy. ${ }^{31}$ Furthermore, individual potassium ions can be visualized by conductive AFM inside the fractals. The locations of potassium ions show a lower conductivity compared to their surrounding because only a $2 \mathrm{D}$-ice layer contributes to charge doping. In the areas surrounding potassium ions, the $2 \mathrm{D}$-ice layer and negatively charged mica surface both contribute to charge doping. In the structural model of the intercalated water developed by other groups, one layer is argued to be located between graphene and mica at ambient conditions. ${ }^{11,35}$ This layer can be partly removed when humidity is reduced. With Raman spectroscopy, they observed the presence of the $D^{\prime}$ peak in low-humidity conditions in areas with removed water layer. They attributed this signal to the ionic mica surface in dry conditions. ${ }^{35}$

When the relative humidity $(\mathrm{RH})$ is lowered to $\sim 0.1 \%$ by purging with nitrogen, part of the top water layer evaporates, ${ }^{11}$ as shown in the schematic illustration in Figure 1a. The molecules leave the graphene-mica slit pore via bottom (B)type step edges. At a B-type step edge, a graphene layer at the bottom of a multilayer graphene flake, which is in direct contact with the water, terminates. The layer above the bottom graphene layer will partly follow the topography of the bottom graphene layer and partly follows the topography of the water molecules on the mica surface. At the location of the B-type step edge, bending of the layer above the bottom graphene layer occurs. At the bending location, a small gap is created in which water molecules can diffuse more easily. An image of a B-type step edge can be found in ref 11 . A $2 \mathrm{D}$-ice layer remains at the location of the evaporated water molecules, with a fractal-like appearance, ${ }^{11,31}$ as shown in the AFM topography image in Figure $1 \mathrm{~b}$. The fractals are darker than their surroundings, indicating a lowering of the graphene height at this location. The fractals are present in an area of monolayer graphene. Figure 1c shows the AFM lateral deflection voltage trace image, which was simultaneously recorded with the AFM topography image. No clear contrast in friction is observed between the $2 \mathrm{D}$-ice and the double water layer, as can also be seen in the inset of Figure 1c. To provide further evidence for this absence of contrast, a zoomed-in friction image will be provided in the next section. Figure $1 \mathrm{~d}$ shows a height profile of the red line in Figure 1b. The depth of the 2D-ice fractal corresponds approximately to a graphene step height (0.34 $\mathrm{nm})$.

When the system is subsequently purged with alcohol, the alcohol molecules fill the region on top of the $2 \mathrm{D}$-ice fractals. ${ }^{36}$ This is schematically depicted in Figure $2 \mathrm{a}$. Figure $2 \mathrm{~b}$ shows the AFM topography image of the same area as in Figure 1b. A small contrast can be discerned, as compared to the double water layer. This contrast is due to the intercalation of 2propanol molecules on top of the $2 \mathrm{D}$-ice fractal. The 2propanol molecules are larger than the water molecules, resulting in an elevation of the graphene sheet. Filling of the 2D-ice fractals typically occurs within tens of minutes ${ }^{36}$ and varies for different locations on the sample due to different local geometries of the graphene. The intercalation of alcohol is observed to be irreversible. The alcohol islands remain when we transfer the sample to ambient. This is in contrast with the observations of Severin et al., ${ }^{20}$ where the alcohol islands shrink when exposed to ambient conditions. Probably, their ambient conditions were under higher humidity, resulting in the intercalation of water molecules back into the slit pore. To test this hypothesis, we exposed the sample to a relative humidity of $80-90 \%$ and we indeed observed that part of the alcohol evaporated from the slit pore and is replaced by water molecules. Figure $2 \mathrm{~d}$ shows the height profile of the red line in Figure 2b. Again an increase in height is observed at the location of the 2-propanol fractal.

Figure $2 c$ shows the AFM lateral deflection voltage trace image, which was simultaneously recorded with the AFM topography image. In this friction image, an increase in friction is observed at the location of the 2-propanol fractal, as compared to the surrounding double water layer. Our proposed explanation for the increase in friction is an increase in the vibrational modes of the intercalated molecules. The alcohol molecules have more vibrational modes compared to the water molecules. The number of vibrational modes is $3 \mathrm{~N}-$ 6 , where $N$ is the number of atoms in the molecule. A water molecule has 3 vibrational modes (bend, symmetric stretch, and asymmetric stretch). Methanol, ethanol, and 2-propanol molecules have already 12,21 , and 30 vibrational modes, respectively. The extra vibrational modes enhance the energy dissipation to the mica. To further validate this assumption, supporting density functional theory calculations should be done. Unfortunately, this lies outside the scope of our work. 

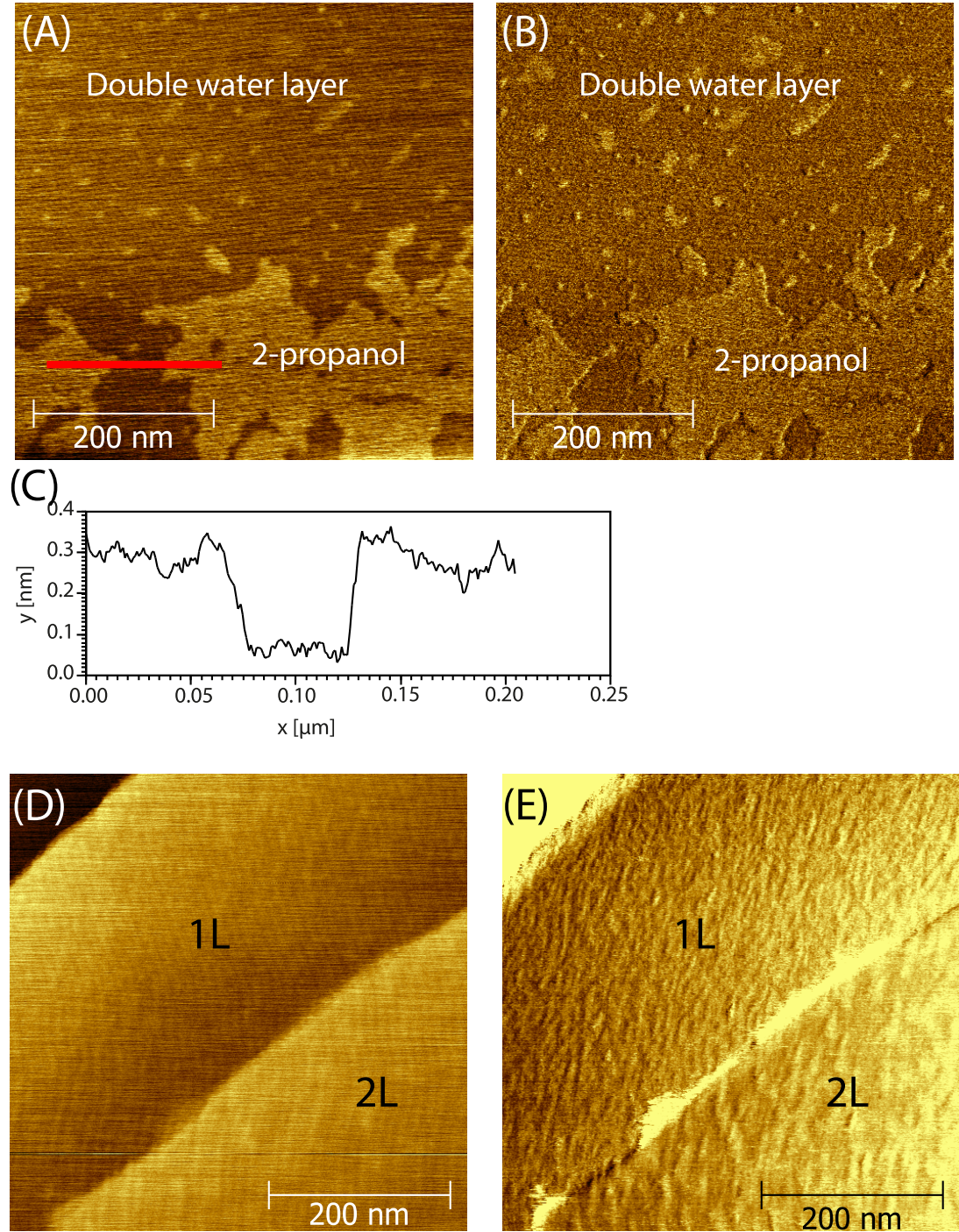

Figure 5. (a) AFM topography image of 2-propanol islands under monolayer graphene. (b) An simultaneously obtained AFM friction image (lateral deflection voltage, trace image). An AD-E-0.5-SS AFM tip was used. (c) Height profile of the red line in (a). (d) An AFM topography image of methanol islands under monolayer graphene. (e) A simultaneously obtained AFM friction image (lateral deflection voltage, trace image). An AD-E-0.5-SS AFM tip was used.

Enhanced puckering of the graphene is not the largest factor for the observed friction increase. Lee et al. ${ }^{8}$ investigated whether puckering could be responsible for an increase in friction of graphene over one and two intercalated water layers. They did this by looking at the strengthening effect of atomic stick-slip friction. The strengthening effect is due to an increased number of atoms that are pinned in perfect synchrony and the increase of pinning. ${ }^{37}$ They found the same strengthening effect in both situations, concluding that the puckering was not responsible for the increase in friction. Replacing a water layer by an alcohol layer will probably have the same influence, since both are liquids under graphene. Furthermore, Lee et al. ${ }^{38}$ showed that puckering can increase the friction of graphene by a factor of 2 . In our measurements with 2-propanol, we observed a factor of 5.9 (to be shown in Figure 5). Therefore, there must be additional contributions to the friction, ascribed to phonon contributions.

Figure 3a presents a zoomed in AFM topography scan of the AFM area of Figure 1b. This location consists of single-layer graphene. The $2 \mathrm{D}$-ice fractal has a lower height compared to the double water layer, as can be seen by the darker contrast in this image. Figure $3 \mathrm{~b}$ shows the simultaneously recorded lateral deflection trace image. No clear contrast in friction is observed, although there is a change in corrugation on the $2 \mathrm{D}$-ice fractals compared to the double water layer. This change in corrugation was already observed by Bampoulis et al. ${ }^{21}$ They attributed the features to the potassium ions on the mica surface. Since graphene perfectly follows the topography of its underlying substrate, individual potassium ions affect the measured LFM image and as such can be visualized. Edge effects are present, but they represent a smaller part of the image and $2 \mathrm{D}$-ice plateaus can be clearly discerned. The absence of contrast in friction between one and two water layers is in contrast with the work of Lee et al. ${ }^{7}$ They observed an increase in friction when comparing one water layer to two water layers. This discrepancy might be due to a different sample preparation method.

To investigate the behavior of a different alcohol, ethanol has been intercalated between graphene and mica in another sample. Figure $4 \mathrm{a}$ shows an AFM topography image of a 
graphene-mica sample with intercalated ethanol. A zoomedout image is provided in the Supporting Information, where the number of graphene layers on mica is more clearly visible. The brighter regions present the intercalated ethanol islands. Like 2-propanol, a higher friction is observed as compared to the double water layer. The number of graphene layers is also indicated. Figure $4 \mathrm{c}$ shows the line profile of the height, corresponding to the red line in Figure 4a. Clear steps in height are observed from the double water to the ethanol islands. A coarsening effect is observed for the ethanol islands under bilayer and trilayer graphene (BLG and TLG, respectively), as compared to SLG. Under SLG, small ethanol islands are visible. Under BLG and TLG, these islands merged together and formed larger alcohol plateaus. This coarsening effect was also found by Severin et al. ${ }^{20,39}$ Figure $4 \mathrm{~b}$ shows the simultaneously recorded lateral deflection trace image of the sample. To observe contrast in the friction between areas with varying number of graphene layers, a significant normal force $F_{\mathrm{N}}$ must be applied to the sample. In this measurement, the applied normal force was $66.4 \mathrm{nN}$. When increasing the normal force, a linear increase in the lateral force $F_{\mathrm{L}}$ was observed, following Amontons' relation $F_{\mathrm{L}}=\mu \mathrm{F}_{\mathrm{N}}{ }^{40}$ where $\mu$ is the friction coefficient.

The magnitude of the friction is significantly larger for SLG as compared to BLG and TLG. We can account for the larger friction for thinner graphene by considering that puckering occurs due to the tip adhesion during sliding of the AFM tip over the surface. The graphene deforms out of plane to ripple in front of the AFM tip in the scanning direction, effectively resulting in a larger contact area. With increasing thickness, the graphene becomes more rigid, i.e., stiffer, which leads to less bending and stretching of the layers. As a result, the puckering is less pronounced and the detected friction is lower. That the friction is higher on SLG is in line with the work of Lee et al. ${ }^{41}$ They observed that the friction of graphene on $\mathrm{SiO}_{2}$ increases with decreasing number of graphene layers. They also found that the same holds for $\mathrm{MoS}_{2}, \mathrm{NbSe}_{2}$, and h-BN on $\mathrm{SiO}_{2} \cdot{ }^{38} \mathrm{Li}$ et al. ${ }^{38,42}$ observed that a change in friction when altering the number of graphene layers does not hold for a mica substrate without intercalated water molecules. This was ascribed to the fact that the graphene strongly adheres to the atomically flat mica. The effect of layer thickness is more pronounced for graphene on ethanol than for graphene on the double water layer. That the friction of graphene on a double water layer is dependent on the layer thickness was already observed by Lee et al. $^{7}$

To quantify the friction, we converted the half-width of the friction loop $W_{\exp }([$ lateral trace - lateral retrace $] / 2)$ to the lateral force $F_{\mathrm{L}}$. In the Supporting Information, the conversion method is explained. Figure $4 \mathrm{~d}$ shows the line profile of the friction, corresponding to the red line in Figure $4 \mathrm{~b}$. A clear stepwise increment in friction is observed as the tip moves from the double water layer to the ethanol islands. For the single-layer graphene on ethanol on top of the $2 \mathrm{D}$-ice layer, the friction is $2.4 \pm 0.14$ times higher than for graphene on top of two layers of water. The bar plot in Figure $4 \mathrm{e}$ summarizes the friction values of Figure $4 \mathrm{~d}$. These values were obtained by taking the mean value of a larger area at the same scan heights. The obtained friction forces are of the same order of magnitude as found in the work of Lee et al.

It is difficult to compare the friction between different alcohols. The AFM tip becomes less sharp after scanning due to forces on the tip and, therefore, the frictional forces change.
The best method is to compare the friction within one scan line or scan lines close to each other. We tried to compare friction by normalizing the friction signal of alcohol to the friction signal of the double water layer, which should be constant. For this comparison, the same number of graphene layers is needed. Figure 5a shows a 2-propanol island under monolayer graphene, and Figure $5 \mathrm{~b}$ shows its corresponding friction image (lateral deflection voltage, trace image). Figure $5 \mathrm{c}$ shows the height profile of the red line in Figure 5a. A friction ratio between the alcohol island and double water layer is measured to be $5.9 \pm 1.0$ (the averaged half-width of the friction loop is taken for the large 2-propanol island and the double water layer). This value is larger than the ratio obtained for ethanol. This larger ratio we ascribe to the additional vibrational modes of the 2-propanol molecule.

Alcohol molecules under monolayer graphene often form small islands, compared to alcohol under multilayer graphene, which preserves its shape. In the Supporting Information, an image is provided in which this graphene layer effect is clearly visible for 2-propanol. The formation of islands is ascribed to a different line tension due to variation in stiffness of the graphene blanket.

For monolayer methanol, however, small alcohol islands $\sim 15 \mathrm{~nm}$ in diameter are always formed. This can be seen in the AFM topography image in Figure $5 \mathrm{~d}$ and in the friction image in Figure 5e (lateral deflection voltage, trace images). In the four samples we tested, we unfortunately always observed small islands under monolayer graphene. Edge effect friction gradients dominate the friction signal of the alcohol islands, which results in a less accurate friction value. For an accurate friction value, large alcohol plateaus are needed. Despite the lower accuracy, a lighter contrast can be observed at the location of the methanol islands, indicating a higher friction. We obtained a friction ratio compared to a double water layer of $1.8 \pm 0.3$ (statistical error).

\section{CONCLUSIONS}

We have studied the influence of alcohol intercalation between graphene and mica on the frictional properties of the graphene. We observed a higher friction for methanol, ethanol, and 2propanol, as compared to an intercalated double water layer, which scales with their size. We attribute this increase in friction to the extra vibrational modes of the alcohol molecules. We also confirmed that single-layer graphene has a higher friction than bilayer and trilayer graphene. To summarize, we demonstrate the ability to tune the friction of graphene by changing the environmental conditions and therewith the intercalated molecules between graphene and mica. Alcohol may therefore be a good candidate to be used in applications in nanodevices where control of the frictional properties of graphene is important.

\section{ASSOCIATED CONTENT}

\section{Supporting Information}

The Supporting Information is available free of charge on the ACS Publications website at DOI: 10.1021/acs.langmuir.9b00471.

Zoomed-out AFM topography image of Figure 4a, AFM topography image of intercalated 2-propanol molecules under different graphene layer thickness, lateral force calibration method (PDF) 


\section{AUTHOR INFORMATION}

\section{Corresponding Authors}

*E-mail: edwin.dollekamp@esrf.fr (E.D.).

*E-mail: h.j.w.zandvliet@utwente.nl (H.J.W.Z.).

\section{ORCID $\odot$}

Edwin Dollekamp: 0000-0001-8956-5785

Pantelis Bampoulis: 0000-0002-2347-5223

\section{Present Addresses}

${ }^{\ddagger}$ II. Physikalisches Institut, Universität zu Köln, Zülpicher Straße 77, 50937 Köln, Germany (P.B.).

${ }^{\dagger}$ European Synchrotron Radiation Facility, 71 Avenue des Martyrs, 38000 Grenoble, France (E.D.).

Notes

The authors declare no competing financial interest.

\section{ACKNOWLEDGMENTS}

This work was supported by the Netherlands Center for Multiscale Catalytic Energy Conversion (MCEC), an NWO Gravitation programme funded by the Ministry of Education, Culture and Science of the government of the Netherlands. P.B. and H.J.W.Z. thank the Stichting voor Fundamenteel Onderzoek der Materie (FOM, FV15714TWDO07) for financial support.

\section{REFERENCES}

(1) Cannara, R. J.; Brukman, M. J.; Cimatu, K.; Sumant, A. V.; Baldelli, S.; Carpick, R. W. Nanoscale Friction Varied by Isotopic Shifting of Surface Vibrational Frequencies. Science 2007, 318, 780783.

(2) Persson, B. N. Sliding Friction: Physical Principles and Applications; Springer Science \& Business Media, 2013.

(3) Carpick, R. W.; Salmeron, M. Scratching The Surface: Fundamental Investigations of Tribology with Atomic Force Microscopy. Chem. Rev. 1997, 97, 1163-1194.

(4) Park, J. Y.; Salmeron, M. Fundamental Aspects of Energy Dissipation in Friction. Chem. Rev. 2014, 114, 677-711.

(5) Zhang, S.; Ma, T.; Erdemir, A.; Li, Q. Tribology of TwoDimensional Materials: From Mechanisms to Modulating Strategies. Mater. Today 2018, DOI: 10.1016/j.mattod.2018.12.002.

(6) Mariani, E.; von Oppen, F. Flexural Phonons in Free-Standing Graphene. Phys. Rev. Lett. 2008, 100, No. 076801.

(7) Lee, H.; Ko, J.-H.; Choi, J. S.; Hwang, J. H.; Kim, Y.-H.; Salmeron, M.; Park, J. Y. Enhancement of Friction by Water Intercalated between Graphene and Mica. J. Phys. Chem. Lett. 2017, 8, 3482-3487.

(8) Lee, H.; Ko, J.-H.; Song, H. C.; Salmeron, M.; Kim, Y.-H.; Park, J. Y. Isotope- and Thickness-Dependent Friction of Water Layers Intercalated Between Graphene and Mica. Tribol. Lett. 2018, 66, 36.

(9) Novoselov, K. S.; Geim, A. K.; Morozov, S. V.; Jiang, D.; Zhang, Y.; Dubonos, S. V.; Grigorieva, I. V.; Firsov, A. A. Electric Field Effect in Atomically Thin Carbon Films. Science 2004, 306, 666-669.

(10) Novoselov, K. S.; Jiang, D.; Schedin, F.; Booth, T. J.; Khotkevich, V. V.; Morozov, S. V.; Geim, A. K. Two-Dimensional Atomic Crystals. Proc. Natl. Acad. Sci. U.S.A. 2005, 102, 1045110453.

(11) Severin, N.; Lange, P.; Sokolov, I. M.; Rabe, J. P. Reversible Dewetting of a Molecularly Thin Fluid Water Film in a Soft Graphene-Mica Slit Pore. Nano Lett. 2012, 12, 774-779.

(12) Lui, C. H.; Liu, L.; Mak, K. F.; Flynn, G. W.; Heinz, T. F. Ultraflat Graphene. Nature 2009, 462, 339-341.

(13) Bunch, J. S.; Verbridge, S. S.; Alden, J. S.; van der Zande, A. M.; Parpia, J. M.; Craighead, H. G.; McEuen, P. L. Impermeable Atomic Membranes from Graphene Sheets. Nano Lett. 2008, 8, 2458-2462.
(14) Lee, C.; Wei, X.; Kysar, J. W.; Hone, J. Measurement of the Elastic Properties and Intrinsic Strength of Monolayer Graphene. Science 2008, 321, 385-388.

(15) Xu, K.; Cao, P.; Heath, J. R. Graphene Visualizes the First Water Adlayers on Mica at Ambient Conditions. Science 2010, 329, $1188-1191$.

(16) Rezania, B.; Dorn, M.; Severin, N.; Rabe, J. Influence of Graphene Exfoliation on the Properties of Water-Containing Adlayers Visualized by Graphenes and Scanning Force Microscopy. J. Colloid Interface Sci. 2013, 407, 500-504.

(17) Nair, R. R.; Blake, P.; Grigorenko, A. N.; Novoselov, K. S.; Booth, T. J.; Stauber, T.; Peres, N. M. R.; Geim, A. K. Fine Structure Constant Defines Visual Transparency of Graphene. Science 2008, 320, 1308.

(18) Gaskell, P. E.; Skulason, H. S.; Rodenchuk, C.; Szkopek, T. Counting Graphene Layers on Glass via Optical Reflection Microscopy. Appl. Phys. Lett. 2009, 94, No. 143101.

(19) Dorn, M.; Lange, P.; Chekushin, A.; Severin, N.; Rabe, J. P. High Contrast Optical Detection of Single Graphenes on Optically Transparent Substrates. J. Appl. Phys. 2010, 108, No. 106101.

(20) Severin, N.; Sokolov, I. M.; Rabe, J. P. Dynamics of Ethanol and Water Mixtures Observed in a Self-Adjusting Molecularly Thin Slit Pore. Langmuir 2014, 30, 3455-3459.

(21) Bampoulis, P.; Sotthewes, K.; Siekman, M. H.; Zandvliet, H. J. W.; Poelsema, B. Graphene Visualizes the Ion Distribution on AirCleaved Mica. Sci. Rep. 2017, 7, No. 43451.

(22) Varenberg, M.; Etsion, I.; Halperin, G. An Improved Wedge Calibration Method for Lateral Force in Atomic Force Microscopy. Rev. Sci. Instrum. 2003, 74, 3362-3367.

(23) Ogletree, D. F.; Carpick, R. W.; Salmeron, M. Calibration of Frictional Forces in Atomic Force Microscopy. Rev. Sci. Instrum. 1996, 67, 3298-3306.

(24) Bampoulis, P.; Lohse, D.; Zandvliet, H. J. W.; Poelsema, B. Coarsening Dynamics of Ice Crystals Intercalated between Graphene and Supporting Mica. Appl. Phys. Lett. 2016, 108, No. 011601.

(25) Ochedowski, O.; Bussmann, B. K.; Schleberger, M. Graphene on Mica - Intercalated Water Trapped for Life. Sci. Rep. 2014, 4, No. 6003.

(26) Shim, J.; Lui, C. H.; Ko, T. Y.; Yu, Y.-J.; Kim, P.; Heinz, T. F.; Ryu, S. Water-Gated Charge Doping of Graphene Induced by Mica Substrates. Nano Lett. 2012, 12, 648-654.

(27) Bampoulis, P.; Sotthewes, K.; Dollekamp, E.; Poelsema, B. Water confined in two-dimensions: Fundamentals and applications. Surf. Sci. Rep. 2018, 73, 233-264.

(28) Rauf, A.; Schilo, A.; Severin, N.; Sokolov, I. M.; Rabe, J. P. Non-monotonous Wetting of Graphene-Mica and $\mathrm{MoS}_{2}$-Mica Interfaces with a Molecular Layer of Water. Langmuir 2018, 34, $15228-15237$.

(29) He, K. T.; Wood, J. D.; Doidge, G. P.; Pop, E.; Lyding, J. W. Scanning Tunneling Microscopy Study and Nanomanipulation of Graphene-Coated Water on Mica. Nano Lett. 2012, 12, 2665-2672.

(30) Lin, H.; Rauf, A.; Severin, N.; Sokolov, I. M.; Rabe, J. P. Influence of Interface Hydration on Sliding of Graphene and Molybdenum-Disulfide Single-Layers. J. Colloid Interface Sci. 2019, 540, 142-147.

(31) Bampoulis, P.; Siekman, M. H.; Kooij, E. S.; Lohse, D.; Zandvliet, H. J. W.; Poelsema, B. Latent Heat Induced Rotation Limited Aggregation in 2D Ice Nanocrystals. J. Chem. Phys. 2015, 143, No. 034702.

(32) Dollekamp, E.; Bampoulis, P.; Poelsema, B.; Zandvliet, H. J. W.; Kooij, E. S. Electrochemically Induced Nanobubbles between Graphene and Mica. Langmuir 2016, 32, 6582-6590.

(33) Dollekamp, E.; Bampoulis, P.; Faasen, D. P.; Zandvliet, H. J. W.; Kooij, E. S. Charge Induced Dynamics of Water in a GrapheneMica Slit Pore. Langmuir 2017, 33, 11977-11985.

(34) Sotthewes, K.; Bampoulis, P.; Zandvliet, H. J. W.; Lohse, D.; Poelsema, B. Pressure-Induced Melting of Confined Ice. ACS Nano 2017, 11, 12723-12731. 
(35) Lin, H.; Schilo, A.; Kamoka, A. R.; Severin, N.; Sokolov, I. M.; Rabe, J. P. Insight into the Wetting of a Graphene-Mica Slit Pore with a Monolayer of Water. Phys. Rev. B 2017, 95, No. 195414.

(36) Bampoulis, P.; Witteveen, J. P.; Kooij, E. S.; Lohse, D.; Poelsema, B.; Zandvliet, H. J. W. Structure and Dynamics of Confined Alcohol-Water Mixtures. ACS Nano 2016, 10, 6762-6768.

(37) Li, S.; Li, Q.; Carpick, R. W.; Gumbsch, P.; Liu, X. Z.; Ding, X.; Sun, J.; Li, J. The Evolving Quality of Frictional Contact with Graphene. Nature 2016, 539, 541.

(38) Lee, C.; Li, Q.; Kalb, W.; Liu, X.-Z.; Berger, H.; Carpick, R. W.; Hone, J. Frictional Characteristics of Atomically Thin Sheets. Science 2010, 328, 76-80.

(39) Severin, N.; Gienger, J.; Scenev, V.; Lange, P.; Sokolov, I. M.; Rabe, J. P. Nanophase Separation in Monomolecularly Thin WaterEthanol Films Controlled by Graphene. Nano Lett. 2015, 15, 11711176.

(40) Amontons, G. De la resistance causee dansles machines. Mém. Acad. R., A 1699, 257-282.

(41) Lee, C.; Wei, X.; Li, Q.; Carpick, R.; Kysar, J. W.; Hone, J. Elastic and Frictional Properties of Graphene. Phys. Status Solidi B 2009, 246, 2562-2567.

(42) Li, Q.; Lee, C.; Carpick, R. W.; Hone, J. Substrate Effect on Thickness-Dependent Friction on Graphene. Phys. Status Solidi B 2010, 247, 2909-2914. 